\title{
Bayeux - 5 rue de la Juridiction
}

Opération préventive de diagnostic et fouille préventive (2013)

\section{Florian Bonhomme}

\section{(2) OpenEdition \\ 1 Journals}

Édition électronique

URL : https://journals.openedition.org/adlfi/16858

ISSN : 2114-0502

Éditeur

Ministère de la Culture

Référence électronique

Florian Bonhomme, «Bayeux - 5 rue de la Juridiction » [notice archéologique], ADLFI. Archéologie de la France - Informations [En ligne], Normandie, mis en ligne le 16 mars 2016, consulté le 08 juin 2021. URL : http://journals.openedition.org/adlfi/16858

Ce document a été généré automatiquement le 8 juin 2021.

(c) ministère de la Culture et de la Communication, CNRS 


\title{
Bayeux - 5 rue de la Juridiction
}

Opération préventive de diagnostic et fouille préventive (2013)

\author{
Florian Bonhomme
}

\section{NOTE DE L'ÉDITEUR}

Organisme porteur de l'opération : Inrap

1 Dans le cadre de la réhabilitation en quatre logements des deux maisons médiévales inscrites, un diagnostic d'archéologie du bâti a été mené à la fin de l'été. Une courte étude a ensuite pu être engagée en fin d'année afin de préciser l'évolution des deux bâtiments. Initialement, ils étaient accessibles depuis la rue Bienvenu, par l'immeuble dit "d'Adam et Ève », lui aussi protégé et déjà restauré. Ces ensembles de bâtiments sont situés au cœur de la ville antique et médiévale de Bayeux, à une trentaine de mètres de la cathédrale, mais aussi en plein cœur du centre du secteur sauvegardé. Ces deux maisons, accolées, sont situées en fond de cour et immédiatement en retrait de l'angle nord-ouest du parvis de la cathédrale.

2 L'évaluation, puis l'étude, ont permis d'appréhender l'imbrication des deux maisons et leur état de conservation. Malgré la présence relativement importante des enduits, l'ensemble des observations archéologiques a mis en évidence un ensemble digne d'intérêt et rarement étudié en élévation en Basse-Normandie. Les deux maisons avaient leur façade qui s'ouvrait chacune sur des cours séparées et n'étaient accessibles que par la rue Bienvenu, située à l'est. Les bâtiments sur la rue leur correspondant étaient les numéros 6 et 8.

Quatre phases principales de constructions et de remaniements ont ainsi été identifiées. La première de ces phases n'exclut pas pour autant la présence de vestiges plus anciens, en particulier en sous-sol, ne serait-ce bien sûr que gallo-romains, ou, plus rarement mis en évidence dans la ville, du haut Moyen Âge.

4 La première construction concerne uniquement les trois premiers niveaux du bâtiment sud, appartenant à la deuxième moitié $d u \mathrm{XII}^{\mathrm{e}} \mathrm{s}$. ou à la première moitié $\mathrm{du} \mathrm{XIII}^{\mathrm{e}} \mathrm{s}$. Les vestiges qui sont conservés sont très importants en dépit de la période à laquelle cette 
maison a été édifiée : les maçonneries du rez-de-chaussée aujourd'hui semi-enterrées et du premier étage. Une dizaine de portes et fenêtres sont conservées dans les maçonneries et permettent de restituer les volumes de manière globale, ainsi qu'une partie des circulations.

5 La seconde période de construction est probablement rattachable au $\mathrm{Xv}^{\mathrm{e}} \mathrm{s}$. Cette phase a vu la reconstruction d'une partie du premier bâtiment sud (pignon nord) et la construction du second, probablement sur les vestiges d'un bâtiment antérieur. Étant donné la porte à mi-niveau du premier bâtiment qui devait correspondre avec le second, l'origine du bâtiment est a priori à rechercher également autour des $\mathrm{XII}^{\mathrm{e}}-\mathrm{XIII}^{\mathrm{e}} \mathrm{s}$. Le bâtiment nord était relié à la maison dite "d'Adam et Ève », en façade de la rue, par une galerie à deux niveaux sur le côté nord de la cour, schéma classique dans l'urbanisme médiéval. L'emplacement de la tour d'escalier qui dessert le second bâtiment est attribué à cette phase. Vraisemblablement durant la deuxième moitié du $\mathrm{XV}^{\mathrm{e}} \mathrm{s}$., des enduits peints ont conclu les travaux. Ces peintures identifiées au rez-dechaussée du bâtiment nord sont d'une grande qualité. Elles ne sont qu'exceptionnellement conservées dans l'habitat civil urbain et sont d'autant plus rares en Basse-Normandie. Ces peintures devaient constituer au moins une frise en partie supérieure et peut-être d'autres décors sur le reste de la pièce. Une autre peinture a été identifiée dans la tour de l'escalier, au niveau d'un linteau de porte, représentant un homme avec un chapeau à plume dans l'encadrement d'une fenêtre, typique de la Renaissance.

6 La troisième phase a de toute évidence vu la reconstruction d'une partie du second bâtiment, au nord, avec en particulier les murs gouttereaux. Les moulures des ouvertures et les décors identifiés permettent d'attribuer ces travaux à la seconde moitié du $\mathrm{XVI}^{\mathrm{e}} \mathrm{s}$. Le pignon sud ne semble pas avoir été concerné. Le pignon nord a quant à lui fait l'objet de quelques reprises ponctuelles en particulier du côté ouest, dans l'angle. La façade orientale a fait l'objet de lourds travaux, puisque plus des trois quarts ont été rebâtis. La façade occidentale a été reprise de part et d'autre de la tour d'escalier avec la création de nouvelles fenêtres à chaque étage. La surélévation du bâtiment sud est attribuable au XVI ${ }^{\mathrm{e}}$ s. également.

7 La dernière période de modifications importantes a eu lieu à la fin du XVII $\mathrm{s}$. ou dans le courant du XVIII $\mathrm{e}$. Elle a eu pour conséquence la redistribution des circulations entre les deux bâtiments, réalisée depuis la tour de l'escalier pour les niveaux supérieurs (premier, deuxième étage et combles). Les percements de portes dans le pignon commun aux deux bâtiments, dont les maçonneries couvrent les derniers badigeons, démontrent qu'ils sont postérieurs au $\mathrm{XvI}^{\mathrm{e}} \mathrm{s}$. Les moulures des cheminées des deux étages viennent confirmer cette attribution chronologique. Les dispositions des cheminées correspondent de plus à la création de divisions dans les pièces de tous les niveaux, à l'aide de cloison de panneaux de bois et de torchis. La dernière a pu être observée au rez-de-chaussée avant sa démolition. Les grandes fissures visibles aujourd'hui sur le pignon sud de la première maison sont peut-être le prolongement des raisons qui ont entrainé la reconstruction d'une importante portion de la partie basse du gouttereau ouest de la maison sud durant cette période de l'Ancien Régime.

Ces deux maisons ont été occupées jusqu'à la Seconde Guerre mondiale. Leur abandon postérieur a entraîné une dégradation importante des toitures et des planchers. Seules les poutres principales des différents niveaux seront conservées, tandis que la 
charpente, refaite depuis environ une décennie, ne présente presque plus aucune pièce ancienne.

Fig. 1 - Détails d'enduits peints de la deuxième moitié du $x v^{e} s$. : un griffon et une femme en robe

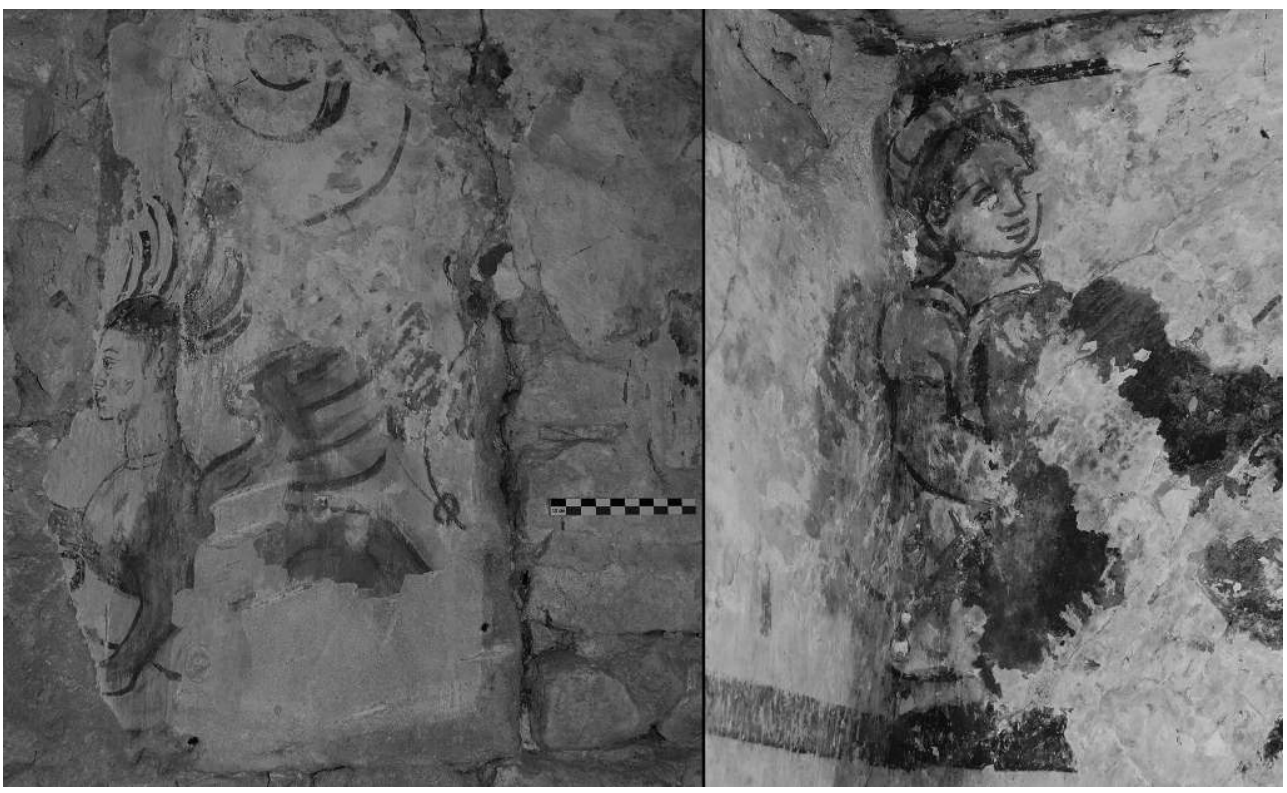

Clichés : F. Bonhomme (Inrap).

\section{INDEX}

Année de l'opération : 2013

nature https://ark.frantiq.fr/ark:/26678/pcrtWWQS75V5Bc, https://ark.frantiq.fr/ark:/26678/ pcrtcJxzOpgs7T

chronologie https://ark.frantiq.fr/ark:/26678/pcrtAQyKm9qosx, https://ark.frantiq.fr/ark:/ 26678/pcrtPSEEZSBEJp

lieux https://ark.frantiq.fr/ark:/26678/pcrtSEeAipsBlD, https://ark.frantiq.fr/ark:/26678/ pcrt85PmfXV4X4, https://ark.frantiq.fr/ark:/26678/pcrtFGjgeNOvS6, https://ark.frantiq.fr/ark:/ 26678/pcrtShBVgb6cQS

\section{AUTEURS}

\section{FLORIAN BONHOMME}

Inrap 